\title{
Intensifying the intensity illusion in judgments of learning: Modality and cue combinations
}

\author{
Zehra F. Peynircioğlu ${ }^{1}$ • Joshua R. Tatz ${ }^{1}$
}

Published online: 8 November 2018

(C) Psychonomic Society, Inc. 2018

\begin{abstract}
We showed that judgments of learning (JOLs) were not affected by presentation modality in a list-learning task, although the typical font-size and loudness illusions emerged in that large-font visual presentations and loud auditory presentations elicited higher JOLs than their less intense counterparts. Further, when items were presented in both modalities simultaneously, largefont/quiet and small-font/loud items received similar JOLs (and were recalled similarly). Most importantly, when the intensity manipulation was compounded across modalities, the magnitude of the illusion increased beyond that observed in a single modality, showing the influence of combining cues. Whereas recall was still the same, large-font/loud items received higher JOLs than either small-font/loud items or large-font/quiet items, and not-intense items received very low JOLs. These differences emerged only when all conditions were presented within a single list and not in a between-subjects design, underscoring the importance of comparative judgments.
\end{abstract}

Keywords Metamemory $\cdot$ Memory $\cdot \mathrm{JOL} \cdot$ Modality $\cdot$ Font size $\cdot$ Loudness

Modality of presentation matters in memory. Echoic memory lasts longer than iconic memory (e.g., Crowder, 1976), and modality and suffix effect findings show sizeable differences between visually and auditorily presented items (e.g., Watkins \& Watkins, 1980). Auditory recoding of visually presented items is favored in short-term memory (e.g., Conrad, 1964), although performance is often better for visually presented items (e.g., Bigelow \& Poremba, 2014). Memory span is affected by presentation modality (e.g., Watkins \& Peynircioğlu, 1983), and working memory models posit separate systems for processing visually and auditorily presented items (e.g., Baddeley \& Hitch, 1974). In long-term memory, a modality match between encoding and retrieval enhances recognition, especially if modality is made a salient attribute or if the retrieval task also relies on the use of perceptual information (e.g., Mulligan, Besken, \& Peterson, 2010; Mulligan \& Osborn, 2009). In short, modality of presentation matters in memory.

Zehra F. Peynircioğlu

peynir@american.edu

1 Department of Psychology, American University, Washington, DC 20016, USA
Whether modality of presentation matters in metamemory has not been researched much, however, possibly because metamemory is often assumed to be a higher-level and more abstract process that should be largely independent of modality. Cue-utilization theory (Koriat, 1997) supports this assumption in that, although people do rely on intrinsic cues that pertain to the item itself in making metamemory decisions, they are less sensitive to the presence of extrinsic cues that pertain to the study situation. Modality of presentation is usually seen as an extrinsic cue and thus is more likely to be discounted or ignored. However, exceptions have been shown (e.g., Carroll \& Korukina, 1999; Peynircioğlu, Brandler, Hohman, \& Knudson, 2014), and Castel (2008) has suggested that extrinsic cues can also affect metamemory decisions if they are made salient.

The primary aim of this study is to further explore presentation modality as a cue for metamemory decisions within the domain of judgments of learning (JOLs) and also look at how information in different cues might be combined to influence such decisions. In the past decade or so, when items are presented in a single modality, other extrinsic-cue manipulations have indeed been shown to influence people's JOLs, but not in a beneficial manner, leading to such phenomena as the fontsize illusion in the visual domain (Rhodes \& Castel, 2008) and the loudness illusion in the auditory domain (Rhodes \& Castel, 2009). People give higher JOLs to items presented in 
a larger font as well as to items presented more loudly, even though they do not actually recall such items any better. In this study, we make use of these illusory findings in posing our questions.

Our first question is whether presenting items visually or auditorily influences JOLs. Because most people often remember visually presented items better than auditorily presented items (e.g., Furnham, Gunter, \& Green, 1990) and appear to be cognizant of this difference (e.g., Krätzig \& Arbuthnott, 2006), we ask whether this knowledge is reflected in their JOLs, as well, showing an effective use of the modality cue. Further, for the same reason, a manipulation in the visual modality (font size) might be more effective than that in the auditory modality (loudness). Similarly, when an item is presented both visually and auditorily simultaneously, just a visual intensity manipulation might be more effective than just an auditory intensity manipulation. Of course, these are the predictions only if the intensity illusion persists in these situations in that any higher JOLs turn out to be unwarranted because recall itself does not vary.

Our second question is how individuals might combine information from two different cues, in this case presentation modality and intensity. In addressing this question, we ask whether font-size and loudness illusions, both of which rely on the manipulations of an intensity cue, are still present or even enhanced when items are presented in both modalities together. That is, if a large-font item is judged to be better learned in visual presentation, and a loud item is judged to be better learned in auditory presentation, will a large-font and loud item be judged to be even better learned than the more intense version in the single modality presentation? Such a result would be predicted both by the perceptual fluency account (e.g., Rhodes \& Castel, 2008) as well as the belief account (e.g., Mueller, Dunlosky, Tauber, \& Rhodes, 2014), the two currently popular accounts proposed to explain the intensity illusions. The perceptual fluency account holds that higher intensity items are processed more readily and are thus thought to be learned better, and the belief account holds that people simply think higher intensity items should be easier to remember. Although the arguments for and against the perceptual fluency account are still ongoing (e.g., Susser, Jin, \& Mulligan, 2016; Yang, Huang, \& Shanks, 2018), of interest for present purposes is that it would predict higher JOLs for a large-font and loud item compared with just a large-font or just a loud item as long as people process the intensity differences in the two modalities during audiovisual integration. The same finding would be predicted by the belief account, as well, as long as people utilize the modality information and think increased presentation intensity in two modalities should lead to better memory than that in a single modality.

Finally, Susser, Mulligan, and Besken (2013) have shown that the font-size effect holds in within-subjects but not between-subjects manipulations, suggesting that relative differences are what participants base their JOLs on. This is consistent with Castel's (2008) suggestion that extrinsic cues matter only if they are made salient. If participants are not exposed to both conditions, the salience created by relative differences is minimized because people can make use of only their generic beliefs rather than actually witnessing the differences, and perceptual fluency differences cannot be compared directly, either. Thus, within this context, we conducted the same experiments using both within-subjects and betweensubjects designs to see if any modality effects would emerge in the former but not the latter type of design. Such differentiation would also inform our second question regarding the utilization of combined information from multiple cues as a function of whether different-intensity and different-modality items are available for direct comparison or not.

In all experiments, participants gave JOL ratings for items presented visually and/or auditorily and in their high-intensity or low-intensity forms. In Experiment 1, participants received large-font words, small-font words, loud words, or quiet words, all intermixed randomly within a single list. In Experiment 2, we replicated Experiment 1 in an all betweensubjects design. In Experiment 3, in a within-subjects design, participants received the words in both modalities coupled together, but sometimes the intensity of the auditory version was exaggerated (i.e., loud presentation but in small font) and sometimes the intensity of the visual version was exaggerated (i.e., large-font but quiet presentation). In Experiments 4 and 5 , we added the two remaining versions of the possible couplings, the not intensified version (i.e., small-font/quiet) as well as the doubly intensified version (i.e., large-font/loud), in a within-subjects design in Experiment 4 and a betweensubjects design in Experiment 5.

\section{Experiment 1}

\section{Method}

Participants A total of 20 American University students participated in the study for extra credit in psychology courses. None reported problems with sight or hearing. Sample sizes for the current and subsequent experiments were determined a priori using power analyses (Faul, Erdfelder, Buchner, \& Lang, 2009). In particular, the experiments were designed to detect medium effect sizes at $\alpha=0.05$ with $80 \%$ power.

Materials The study was modeled after the Rhodes and Castel (2008, 2009) studies. The presentation list comprised 40 nouns selected from those used in these studies. All stimuli were presented on a Toshiba Satellite C55-C computer with a 39.6-cm diameter display and $1366 \times 768$ pixels resolution using Microsoft PowerPoint. Each word was placed on a different slide that was set to advance automatically after $5 \mathrm{~s}$. For 
the visual conditions, words were written in the standard 18-pt (small) or 48-pt (large) Ariel fonts and displayed in the center of the screen. Participants were seated approximately $65 \mathrm{~cm}$ away from the screen. For the auditory conditions, the original quiet or loud recordings from Rhodes and Castel's study (2009) were imported from .wav files onto each slide and set to play automatically. The loudness levels were approximately $65 \mathrm{~dB}$ sound pressure level (SPL) for quiet presentations and $75 \mathrm{~dB}$ SPL for loud presentations, with ambient noise at about $30 \mathrm{~dB}$ SPL. Each word was approximately $0.5-1.0 \mathrm{~s}$ in duration and was played only at the start of its presentation slide that was blank except for a white fixation cross in the center. There was also a rating screen containing the scale 1 (not at all likely) 100 (very likely), which was displayed after each presentation screen and automatically advanced after the prescribed time. Paper and pencil were used to record the JOL ratings and recall responses.

Design and procedure The presentation list comprised 40 words, with the first two and the last two words serving as the primacy and recency buffers, and the remaining 36 as the main targets. These 36 words were divided into two sets of 18 , one set to be presented visually (with a subset of nine presented in large font and the other subset of nine in small font) and the other set to be presented auditorily (with nine presented loudly and the other nine quietly). The words were then randomly intermixed with respect to these four conditions. Although the presentation order was the same for all participants, each set of nine words was rotated across four groups of participants so that each word served in each of the modality and intensity conditions equally often.

Participants were tested individually. During the presentation phase, they studied the words for a subsequent memory test and made a JOL after each word. Each word was studied for $5 \mathrm{~s}$. Sometimes the word was presented on the screen, in which case participants looked at it for as long as it was in view, and sometimes the screen was blank except for a fixation cross, and the word was spoken, in which case participants listened carefully and were asked to keep hearing it in their heads until the next screen. Sometimes the written words were large and sometimes small, and, similarly, sometimes the spoken words were loud and sometimes quiet. In between each study word was the JOL screen, which was on for $4 \mathrm{~s}$, during which time participants indicated on a sheet of paper how well they had learned the word they had just studied on a scale of 1 to 100 . The JOL screen disappeared $1 \mathrm{~s}$ before the next study screen appeared. Before the main experiment, participants practiced the procedure, in which they were exposed to all four conditions and the JOL screens using the word sample.

After the presentation phase, participants were given a 5min distractor task in which they wrote down as many U.S. states and, if they ran out of them, as many countries as they could. Finally, a 4-min free-recall test followed in which the task was to write down as many of the studied words as they could remember in any order they wished.

\section{Results}

The results are presented in Table 1. A 2 (intensity: high vs. low) $\times 2$ (presentation modality: auditory vs. visual) repeatedmeasures ANOVA showed a main effect of intensity on mean JOL ratings such that higher intensity study items received higher JOLs, $F(1,19)=34.54, M S E=83.42, p<.01, \eta_{p}^{2}=$ .65. Further, post hoc pairwise comparisons showed that the effect of intensity was present in each modality separately, as well, with loud sounds and large fonts receiving higher JOLs than their quieter and smaller versions, $t \mathrm{~s}(19)=4.35$ and 5.84 , $p \mathrm{~s}<.001$, and $d \mathrm{~s}=0.97$ and 1.30 , respectively. There was, however, no main effect of modality on JOLs, $F(1,19)=1.39$, $M S E=28.73, p=.25$, nor an interaction between intensity and modality, $F(1,19)=0.20, M S E=125.41, p=.66$.

More importantly, recall performance was unaffected by intensity or modality, $F_{\mathrm{s}}(1,19)=0.12$ and $0.14, M S E \mathrm{~s}=$ 117.85 and $272.50, p s=.74$ and .71 , and neither was there an intensity by modality interaction, $F(1,19)=0.25, M S E=$ $303.04, p=.62$. Thus, the higher JOL ratings given to higher intensity items were not warranted in either the visual or the auditory mode of presentation. ${ }^{1}$ Both the font size and the loudness illusions remained intact, suggesting that the participants were able to segregate within-modality comparisons in making their judgments, and modality itself did not seem to be used as a separate cue that influenced these judgments.

\section{Experiment 2}

We replicated Experiment 1 but used a between-subjects design to remove the influence of direct comparisons in terms of both modality and intensity. The purpose was to decrease the salience of these cues to see if any effects of intensity leading to an illusion would disappear, especially in the auditory modality, as loudness has not been tested in such a design yet.

\footnotetext{
${ }^{1}$ We should note that JOL accuracy analyses corroborated the finding that increased JOLs for high-intensity items were illusory and led to less accurate predictions. The usual Kruskal-Goodman gamma correlations could not be used because of the relatively small number of observations in each condition. Instead, we computed alternative accuracy scores (cf. Rabinovitz \& Peynircioğlu, 2011), whereby, in each condition, the number of recalled items that had received lower JOLs $(<50)$ were combined with the number of unrecalled items that had received higher JOLs $(>51)$ and then subtracted from the number of recalled items that had received higher JOLs combined with the number of unrecalled items that had received lower JOLs $(<50)$. Thus, the scores varied from -9 (greatest inaccuracy) to 9 (greatest accuracy). There was a main effect of intensity, $F(1,19)=10.19, M S E=9.93, p=.005, \eta_{p}^{2}=$ .35 , but no main effect of modality, $F(1,19)=0.00, M S E=16.26, p=$ .96 , nor an interaction between intensity and modality, $F(1,19)=0.67$, $M S E=9.05, p=.42$.
} 
Table 1 Mean JOL ratings (1-100) and mean percentage recalled as a function of presentation modality and intensity in Experiments 1 and 2 (standard deviations are in parentheses)

\begin{tabular}{llllll}
\hline & \multicolumn{2}{c}{ Auditory presentation } & & \multicolumn{2}{l}{ Visual presentation } \\
\cline { 2 - 3 } \cline { 5 - 6 } \cline { 5 - 6 } & Low intensity & High intensity & & Low intensity & High intensity \\
\hline Experiment 1 & & & & \\
JOL & $47.5(15.7)$ & $58.1(17.5)$ & & $47.2(17.7)$ & $60.7(20.1)$ \\
Recall & $20.0(19.9)$ & $19.4(14.4)$ & & $17.2(13.7)$ & $20.6(22.0)$ \\
Experiment 2 & & & & & \\
JOL & $45.6(15.0)$ & $55.1(13.6)$ & & $47.2(12.2)$ & $53.0(13.2)$ \\
Recall & $16.1(6.3)$ & $21.7(13.2)$ & & $25.0(10.0)$ & $23.5(10.3)$ \\
\hline
\end{tabular}

\section{Method}

Participants A total of 80 American University students participated in the study for extra credit in psychology courses. None had participated in Experiment 1, and none reported problems with sight or hearing.

Materials, design, and procedure The method was identical to that of Experiment 1 except that one group of participants received all 40 words in the small-font visual presentation condition, another group received them in the large-font visual presentation condition, another group received them in the loud-auditory presentation condition, and another group received them in the quiet-auditory presentation condition. Thus, there were again two high-intensity conditions, one visual and one auditory, and two low-intensity conditions, one visual and one auditory. But this time, the JOLs were made without the benefit of direct comparisons between the different conditions.

\section{Results and discussion}

The results are presented in Table 1. A 2 (intensity: high vs. low) $\times 2$ (presentation modality: auditory vs. visual) ANOVA was conducted. There was a main effect of intensity in JOL ratings, $F(1,76)=6.36, M S E=183.42, p=.01, \eta_{p}^{2}=.08$. There was, however, no main effect of modality, $F(1,76)=$ $0.01, M S E=183.42, p=.92$, nor an interaction between intensity and modality, $F(1,76)=0.38, M S E=183.42, p$ $=.54$.

Recall performance was unaffected by intensity, $F(1,76)=$ $0.77, M S E=105.13, p=.38$, neither in the auditory modality, $t(38)=1.70, p=.10$, nor in the visual modality, $t(38)=0.48, p$ $=.64$. But it was affected by modality, $F(1,76)=5.44, M S E=$ $105.13, p=.02, \eta_{p}^{2}=.07$. Visually presented items were recalled better than auditorily presented items. There was no interaction between presentation modality and intensity, $F(1$, 76) $=2.39, M S E=105.13, p=.13$.
The finding that recall appeared to be unaffected by intensity suggests that the difference found in JOL ratings with auditorily presented items was again likely illusory. ${ }^{2}$ Further, the finding that recall was better for visually presented items than for auditorily presented items was consistent with previous findings in the memory literature (e.g., Furnham et al., 1990). Coupled with this finding, the finding that JOL ratings did not differ as a function of modality could be interpreted as showing yet another dissociation between memory and metamemory processes (e.g., Besken \& Mulligan, 2013). However, we think it is more likely that the JOL findings showed that participants' decisions were influenced primarily by relative comparisons within a single list (comprising all same-modality items) rather than showing unawareness of the memory advantage for visual items in general. We should also note that this memory advantage was not present in Experiment 1, possibly because when the list was mixed in terms of modality, engaging in the JOL task drew attention to the actual learning and participants were able to compensate for the influence of modality differences.

\section{Experiment 3}

In this experiment, we combined the visual and auditory presentations of each word to see whether intensifying just the visual modality while keeping the auditory modality quiet would lead to different JOLs as well as a different memory performance compared with intensifying just the auditory modality while keeping the visual modality in small font. Of interest was whether presenting both modalities at the same time would draw attention to the modality cue and affect the intensity illusion differentially in the two conditions, or whether presentation modality would be discounted as a cue as in the previous two experiments.

\section{Method}

Participants A total of 20 American University students participated in the study for extra credit in psychology courses. None had participated in the previous experiments, and none reported problems with sight or hearing.

Materials, design, and procedure The method was the same as in Experiment 1, except that each word was presented both visually and auditorily simultaneously, there were only two presentation conditions, and the numbers of primacy and recency buffer words were increased to three each (cf.

\footnotetext{
${ }^{2}$ As in Experiment 1, accuracy analyses, computed in the same way, showed a main effect of intensity, $F(1,76)=5.70, M S E=136.08, p=.02, \eta_{p}^{2}=.07$, but not of modality, $F(1,76)=0.00, M S E=136.08, p=1.00$, nor an interaction between intensity and modality, $F(1,76)=0.41, M S E=$ $136.08, p=.52$.
} 
Rhodes \& Castel, 2008). One of the 18-word sets was presented in large font and quietly, and the other was presented in small font and loudly. These two types of words were intermixed randomly during presentation, and, across two groups of participants, each word was presented equally often in both conditions.

\section{Results and discussion}

The results are presented in Table 2. There was no difference in mean JOL ratings between the two conditions, $t(19)=0.52, p=.61$, and neither was there a difference in recall performance, $t(19)=0.32, p=.75$. Modality of presentation, or more specifically, the relative salience in terms of intensity as a function of modality (large or loud), did not appear to matter. However, this may have been because the two other possible types of presentations (i.e., small-font/quiet and large-font/loud) were missing, and thus participants did not have the explicit anchor points to help them judge (albeit erroneously) the relative benefits of intensity increases in each modality. We included these remaining conditions in Experiment 4 to see whether the current results would be replicated in the presence of these anchor points, and, more importantly, to see whether both the font-size and the loudness illusions would persist in such simultaneous presentation cases. Further, one interesting possibility was that not only would the intensity illusions persist, but a simultaneously intensified item in both modalities would lead to an even a greater illusion than in either modality alone, suggesting evidence for integration of information from both cues in JOLs.

\section{Experiment 4}

\section{Method}

Participants A total of 20 American University students participated in the study for extra credit in psychology courses. None had participated in the previous experiments, and none reported problems with sight or hearing.

Materials, design, and procedure The materials were the same as those used in the previous experiments. They were divided into four sets as in Experiment 1 to serve in the four conditions of interest: large-font/loud (doubly intense), small-font/loud (auditory intense), large-font/quiet (visual intense), and small-font/quiet (not intense). The same counterbalancing measures were taken as in Experiment 1, and the procedure was also the same, except that during the presentation phase, all words were presented both visually and auditorily at the same time.

\section{Results and discussion}

The results are presented in Table 2. As expected, in terms of JOL ratings, a 2 (auditory intensity: high vs. low) $\times 2$ (visual intensity: high vs. low ) repeated-measures ANOVA showed a main effect of auditory intensity, $F(1,19)=14.58, M S E=$ $148.15, p=.001, \eta_{p}^{2}=.43$, a main effect of visual intensity, $F(1,19)=41.02, M S E=265.68, p<.001, \eta_{p}^{2}=0.68$, but no interaction between auditory and visual intensity $F(1,19)=$ $.07, M S E=36.83, p=.80$.

With no such interaction, the intensity effect appeared to be an additive one across modalities. Thus, we conducted a oneway repeated-measures ANOVA to explore in more detail the effects of the intensity manipulation in a single modality or in both modalities together, the main focus of the present experiment. The Holm-Bonferroni method was used to control the family-wise error rate for post hoc comparisons. Since Mauchly's test indicated violation of the sphericity assumption, $\chi^{2}(3)=.38, p=.004$, degrees of freedom were corrected using Greenhouse-Geisser estimates $(\varepsilon=.68)$. There was a main effect of condition for JOL ratings, $F(2.03,12.88)=$ 22.09, $M S E=150.22, p<.001, \eta_{p}^{2}=.54$. The results of Experiment 3 were replicated, in that the ratings were not different as a function of which modality was intensified, $t(19)=0.89, p=.38$. However, the anchor points did appear to be rated differently. Items in the doubly intense condition were given higher JOLs than those in any of the other conditions, $t \mathrm{~s}(19)=6.28,3.57,6.15$, compared with the auditoryintense, visual-intense, and not-intense conditions, respectively, all $p \mathrm{~s}<.01, d \mathrm{~s}>.18$. And the items in the not-intense condition were given lower JOLs than those in both the auditory-intense and visual-intense conditions $t \mathrm{~s}(15)=3.59$ and 5.27, respectively, $p s<.01, d \mathrm{~s}>.18$. There was no effect of condition on recall performance, $F(3,19)=0.63, M S E=$ $113.74, p=.60$, and the assumption of sphericity was not violated, $\chi^{2}(3)=.92, p=.93$.

Thus, compared with the not-intense control condition, when presentation in one or both modalities was intensified, JOLs were higher without an accompanying difference in recall performance. ${ }^{3}$ The illusions emerged. Further, whereas the modality that was manipulated did not matter, when both

\footnotetext{
${ }^{3}$ Accuracy scores were computed as in the previous experiments. Mauchly's test indicated violation of sphericity, $\chi^{2}(3)=.43, p=.01$, and degrees of freedom were corrected using Greenhouse-Geisser estimates $(\varepsilon=.71)$. There was an effect of condition on accuracy, $F(2.12,13.44)=8.29, M S E=$ 17.71, $p<.001, \eta_{p}^{2}=.51$, and post hoc comparisons paralleled those of JOL magnitudes. Accuracy did not differ based on which modality was intensified, $t(19)=.45, p=.66$, but was lower for items in the doubly intense condition, compared with the auditory-intense, visual-intense, and not-intense conditions, $t \mathrm{~s}(19)=3.34,2.76,4.22$, respectively, all $p \mathrm{~s}$ $<.05$. Items in the not-intense condition were judged more accurately than those in both the auditory-intense, and the visual-intense condition, $t \mathrm{~s}(19)=2.59$ and 2.53 , respectively, $p \mathrm{~s}<.05$.
} 
Table 2 Mean JOL ratings (1-100) and mean percentage recalled as a function of presentation modality and intensity in Experiments 3, 4, and 5 (standard deviations are in parentheses).

\begin{tabular}{|c|c|c|c|c|}
\hline & No intensity & Auditory intensity & Visual intensity & Double intensity \\
\hline \multicolumn{5}{|c|}{ Experiment 3} \\
\hline $\mathrm{JOL}$ & - & $57.6(12.0)$ & $57.9(16.1)$ & - \\
\hline Recall & - & $13.6(7.5)$ & $12.8(9.2)$ & - \\
\hline \multicolumn{5}{|c|}{ Experiment 4} \\
\hline $\mathrm{JOL}$ & $39.2(18.0)$ & $53.1(17.3)$ & $55.2(18.3)$ & $70.0(18.7)$ \\
\hline Recall & $14.6(15.2)$ & $12.9(13.0)$ & $14.0(11.6)$ & $17.5(11.3)$ \\
\hline \multicolumn{5}{|c|}{ Experiment 5} \\
\hline JOL & $56.6(16.6)$ & $51.5(22.9)$ & $50.7(19.5)$ & $53.3(12.2)$ \\
\hline Recall & $20.0(8.4)$ & $20.0(9.0)$ & $19.4(12.0)$ & $18.8(7.6)$ \\
\hline
\end{tabular}

were manipulated together, the illusion was even more magnified. These results suggest that although participants were aware of the presence of the different modalities in that they were integrating the information, they did not make use of modality information, and intensity appeared to be the only cue that was utilized, albeit unwarrantedly.

\section{Experiment 5}

In Experiment 4, the unusually low JOLs in the not-intense condition and the unusually high JOLs in the doubly intense conditions could have been due to the relative differences in perceptual fluency or to the bolstering of beliefs about the relationship between one's memory and intensity through direct comparisons between the different conditions in the same list. In Experiment 5, we used a between-subjects design that would eliminate potential relative perceptual fluency differences.

\section{Method}

Participants A total of 80 American University students participated in the study for extra credit in psychology courses. None had participated in the previous experiments, and none reported problems with sight or hearing.

Materials, design, and procedure The method was identical to that of Experiment 4 except that one group of participants received all of the words in the auditory-intense condition, another group received them in the visual-intense condition, a third group received them in the doubly intense condition, and a fourth group received them in the not-intense condition.

\section{Results and discussion}

The results are presented in Table 2. A 2 (auditory intensity: high vs. low) $\times 2$ (visual intensity: high vs. low) ANOVA showed no main effects, $F(1,76)=0.09, M S E=332.69, p=$ .77 , for auditory intensity, and $F(1,76)=0.26, M S E=332.69$, $p=.61$, for visual intensity. There was also no interaction, $F(1$, $76)=.91, M S E=332.69, p=.34$. As in Experiment 4, a oneway ANOVA also showed no differences in JOLs between the four conditions, $F(3,76)=0.42, M S E=332.69, p=.74$. And recall performances were not different, $F(3,76)=0.08, M S E=$ $88.75, p=.97$. Thus, in the absence of opportunity for relative comparisons, the illusions, along with the unusually low JOLs in the not-intense condition and the compounded illusion in the doubly intense condition disappeared.

With these findings, perhaps one point that needs to be addressed is why we had obtained an intensity effect in a similar between-subjects manipulation in Experiment 2. In that experiment, although the interaction between modality and intensity had not been significant, the intensity effect had appeared to be driven primarily by the results in the auditory modality, $t(38)=2.10, p=.04, d=.64$, and, though in the same direction, not quite reached significance in the visual modality, $t(38)=1.43, p=.16$. In the current experiment, possibly because both modalities were present simultaneously, the significant main effect of JOL intensity disappeared with an apparent takeover from visual modality. This is consistent with multisensory perception literature suggesting that information across sensory channels may be combined with nonequal weightings that generally favor visual contributions (e.g., McGurk \& MacDonald, 1976). That is, if the independent reliability (i.e., the inverse of its variance) of a perceptual judgment (e.g., intensity) for one sense is much greater than that in an additional sense, the resulting bimodal percept might not differ from that produced by the more reliable sense (e.g., Ernst \& Banks, 2002). Using such perceptual measures, it may be interesting to see whether any effects in perception carry over to systems like memory and metamemory. Of course, we cannot speak as to whether, in this case, the visual sense was the more reliable one, although this might be suggested by the higher recall for 
the visual-only items in Experiment 2. Within this framework, a future study might explore the possibility of asymmetric integration of modality information in metamemory by systematically varying the intensity levels of one modality, and then examining whether the inclusion of information from a second modality influences JOLs and recall similarly at all intensity levels of the first modality. This could also inform the fluency versus beliefs debate, as the former might predict that adding auditory information increases JOLs and memory most at relatively low visual intensities whereas the latter might predict dissociated memory and JOLs (cf. Ross, Saint-Amour, Leavitt, Javitt, \& Foxe, 2006).

\section{General discussion}

We showed that modality of presentation did not influence JOLs directly. Participants gave similar ratings to visually presented items as they did to auditorily presented items, even when in Experiment 2, they recalled visually presented items better. Further, both the font-size and loudness illusions (Rhodes \& Castel, 2008, 2009) were replicated in the same experiment, and the magnitudes of the illusions were similar (although equating the intensity differences in the two modalities was not possible), suggesting again that modality of presentation did not matter.

Interestingly, in Experiment 2, the intensity illusion remained even in a between-subjects design, although the effect appeared to be somewhat stronger in the auditory than in the visual modality. We speculate that perhaps the loud or quiet items we used were comparatively more distinct from what individuals encounter day to day, and this perceptual oddity made the loudness manipulation more salient than the font-size manipulation in this particular task. That is, the different font sizes we used may have fallen more within what is known as the "fluent range" (e.g., Legge \& Bigelow, 2011; Undorf, Zimdahl, $\&$ Bernstein, 2017) compared with the variations in the loudness dimension. The reason for why between-subjects manipulations have not shown the intensity illusion to date (e.g., Susser et al., 2013) could then be because, in the absence of direct relative comparisons, the presented intensities may not have crossed threshold to make them seem like useful cues.

More interestingly, in answering the main question in this paper, even though participants were not using modality information in making JOLs directly, the emergence of the compounded illusion nevertheless suggested that JOLs were based on information integrated from both modalities. When items were presented visually and auditorily simultaneously, doubly intense presentations led to a greater illusion compared with increased intensity in only one of the modalities. Thus, these intensity illusions appeared to arise only after the intensity information was integrated from the two different modalities, supporting the assumption that metamemory decisions do in fact involve a higher level of processing. Such an assumption could also at least partly explain why extrinsic cues pertaining to the study situation rather than the item itself are often discounted (Koriat, 1997) because such cues are more relevant at the lower stages of processing and are already in integrated form when a metamemory decision is made. At the very least, it appears then that multiple cues can in fact be combined in making metacognitive decisions (e.g., Undorf, Söllner, \& Bröder, 2018), even when one of the cues may not be utilized at all when presented separately.

Of course, whether participants considered the intensity contributions of each modality individually before considering the contribution of their intensity in an integrated form is unknown. One potential means of assessing this in the future might involve seeing whether any source information is retained at the time of recall. That is, if a participant remembered a given word, could he or she also remember whether that source was visually or auditorily intense? If so, it would suggest that unimodal intensities were in fact noticed, but neither modality was thought to be more advantageous than the other.

The present findings can be explained by both the perceptual fluency (Rhodes \& Castel, 2008) and the belief (Mueller et al., 2014) accounts. However, the limitation of the compounded illusion to just the within-subjects manipulation underscores the importance of relative comparisons in the emergence of such metamemory differences, whether it is because true differences in perceptual fluency are needed to calibrate these JOLs or because beliefs need to be reminded or reinforced for them to play a substantial enough role. A similar conclusion is reached when we look at the JOLs for items in the not-intense condition. It is either relative perceptual fluency or reinforced beliefs that are likely to bring down the JOLs for these items to the unusually low levels observed when direct comparisons are available from the typically higher JOLs they receive when they are the only items being rated.

Author note We thank Matthew Rhodes and Alan Castel for sharing their materials and giving feedback on the initial idea. We thank José Muniz for his help in Experiments 2 and 5. We also thank an anonymous reviewer for suggesting the first part of the title. Correspondence concerning this article can be addressed to Zehra F. Peynircioğlu, Department of Psychology, American University, Washington, DC, 20016, or peynir@american.edu

Publisher's Note Springer Nature remains neutral with regard to jurisdictional claims in published maps and institutional affiliations. 


\section{References}

Baddeley, A. D., \& Hitch, G. (1974). Working memory. Psychology of Learning and Motivation, 8, 47-89.

Besken, M., \& Mulligan, N. W. (2013). Easily perceived, easily remembered? Perceptual interference produces a double dissociation between metamemory and memory performance. Memory \& Cognition, 41, 897-903.

Bigelow, J., \& Poremba, A. (2014). Achilles' ear? Inferior human shortterm and recognition memory in the auditory modality. PLOS ONE, 9(2), e89914.

Carroll, M., \& Korukina, S. (1999). The effect of text coherence and modality on metamemory judgements. Memory, 7(3), 309-322.

Castel, A. D. (2008). Metacognition and learning about primacy and recency effects in free recall: The utilization of intrinsic and extrinsic cues when making judgments of learning. Memory \& Cognition, $36(2), 429-437$.

Conrad, R. (1964). Acoustic confusions in immediate memory. British Journal of Psychology, 55(1), 75-84.

Crowder, R. G. (1976). Principles of learning and memory. Hillsdale, NJ: Erlbaum.

Ernst, M. O., \& Banks, M. S. (2002). Humans integrate visual and haptic information in a statistically optimal fashion. Nature, 415(6870), 429-433.

Faul, F., Erdfelder, E., Buchner, A., \& Lang, A. G. (2009). Statistical power analyses using $\mathrm{G}^{*}$ Power 3.1: Tests for correlation and regression analyses. Behavior Research Methods, 41(4), 1149-1160.

Furnham, A., Gunter, B., \& Green, A. (1990). Remembering science: The recall of factual information as a function of the presentation mode. Applied Cognitive Psychology, 4(3), 203-212.

Koriat, A. (1997). Monitoring one's own knowledge during study: A cueutilization approach to judgments of learning. Journal of Experimental Psychology: General, 126(4), 349-370.

Krätzig, G. P., \& Arbuthnott, K. D. (2006). Perceptual learning style and learning proficiency: A test of the hypothesis. Journal of Educational Psychology, 98(1), 238-246.

Legge, G. E., \& Bigelow, C. A. (2011). Does print size matter for reading? A review of findings from vision science and typography. Journal of Vision, 11(5), 1-22.

McGurk, H., \& MacDonald, J. (1976). Hearing lips and seeing voices. Nature, 264(5588), 746.

Mueller, M. L., Dunlosky, J., Tauber, S. K., \& Rhodes, M. G. (2014). The font-size effect on judgments of learning: Does it exemplify fluency effects or reflect people's beliefs about memory? Journal of Memory and Language, 70, 1-12.

Mulligan, N. W., Besken, M., \& Peterson, D. (2010). Remember-know and source memory instructions can qualitatively change old-new recognition accuracy: The modality-match effect in recognition memory. Journal of Experimental Psychology: Learning, Memory, and Cognition, 36(2), 558-566.

Mulligan, N. W., \& Osborn, K. (2009). The modality-match effect in recognition memory. Journal of Experimental Psychology: Learning, Memory, and Cognition, 35(2), 564-571.

Peynircioğlu, Z. F., Brandler, B. J., Hohman, T. J., \& Knutson, N. (2014). Metacognitive judgments in music performance. Psychology of Music, 42(5), 748-762.

Rabinovitz, B. E., \& Peynircioğlu, Z. F. (2011). Feeling-of-knowing for songs and instrumental music. Acta Psychologica, 138(1), 74-84.

Rhodes, M. G., \& Castel, A. D. (2008). Memory predictions are influenced by perceptual information: Evidence for metacognitive illusions. Journal of Experimental Psychology: General, 137(4), 615625.

Rhodes, M. G., \& Castel, A. D. (2009). Metacognitive illusions for auditory information: Effects on monitoring and control. Psychonomic Bulletin \& Review, 16(3), 550-554.

Ross, L. A., Saint-Amour, D., Leavitt, V. M., Javitt, D. C., \& Foxe, J. J. (2006). Do you see what I am saying? Exploring visual enhancement of speech comprehension in noisy environments. Cerebral Cortex, 17(5), 1147-1153.

Susser, J. A., Jin, A., \& Mulligan, N. W. (2016). Identity priming consistently affects perceptual fluency but only affects metamemory when primes are obvious. Journal of Experimental Psychology: Learning, Memory, and Cognition, 42(4), 657-662.

Susser, J. A., Mulligan, N. W., \& Besken, M. (2013). The effects of list composition and perceptual fluency on judgments of learning (JOLs). Memory \& Cognition, 41(7), 1000-1011.

Watkins, M. J., \& Peynircioğlu, Z. F. (1983). Interaction between presentation modality and recall order in memory span. The American Journal of Psychology, 96(3), 315-322. https://doi.org/10.2307/ 1422314

Watkins, O. C., \& Watkins, M. J. (1980). Echoic memory and voice quality: Recency recall is not enhanced by varying presentation voice. Memory \& Cognition, 8(1), 26-30.

Undorf, M., Söllner, A., \& Bröder, A. (2018). Simultaneous utilization of multiple cues in judgments of learning. Memory \& Cognition, 46(4), 507-519.

Undorf, M., Zimdahl, M. F., \& Bernstein, D. M. (2017). Perceptual fluency contributes to effects of stimulus size on judgments of learning. Journal of Memory and Language, 92, 293-304.

Yang, C., Huang, T. S. T., \& Shanks, D. R. (2018). Perceptual fluency affects judgments of learning: The font size effect. Journal of Memory and Language, 99, 99-110. 